\title{
Severe metabolic bone disease in pregnancy mimicking malignancy
}

\author{
Jennifer S. Ringrose MD MSc, Ho Jen MD, Carolyn O'Hara MD, Ellen Toth MD
}

A 21-year-old woman of El Salvadorian descent (born in El Salvador, with immigration to Canada at age three years), presented with a deformity of the right humerus in December, thought to have occurred following minor trauma. Radiography showed a fracture. She had a positive result on a pregnancy test in January. She experienced abdominal pain the next month, and a ureteric stone was diagnosed. At that time, alkaline phosphatase was 1434 (normal 30-130) $\mathrm{U} / \mathrm{L}$; serum calcium was not measured. In June, when the patient underwent follow-up radiography for the previously reported fracture, the image showed an aggressive-appearing lytic lesion in the proximal right humerus (Figure 1). Magnetic resonance imaging (MRI) showed numerous other bone lesions involving the scapula and ribs. The scapular and humeral lesions (Figure 2) were sampled for biopsy, and the pathology report suggested giant cell tumour of bone. In September, after delivering a baby that was small for gestational age but otherwise healthy, the patient underwent follow-up imaging with MRI, plain radiography and bone scanning. The findings of these investigations were suggestive of metabolic bone disease, with multiple brown tumours in both the axial and appendicular skeleton (Figure 3), rather than giant cell tumour. No treatment was initiated at that time.

Four months after the delivery, the patient was admitted to hospital following a motor vehicle collision. A minimally displaced pelvic fracture was suspected on pelvic radiography. However, the trauma of the collision was minor, and it was unclear whether the lesion seen radiographically was a fracture or a manifestation of the previously suspected metabolic bone disease. Serum calcium was elevated, at 3.43 (normal $2.10-2.60$ ) $\mathrm{mmol} / \mathrm{L}$, as were alkaline phosphatase (1046 [normal 30130] U/L), parathyroid hormone (244.4 [normal 1.1-6.8] pmol/L [1 pmol/L $=9.49 \mathrm{ng} / \mathrm{L}]$ ) and urine calcium (29.4 [normal 2.0-7.5] mmol/day). Serum 25-hydroxyvitamin D was reduced, at 9 (normal 80-200) nmol/L. Serum albumin was within normal limits. Although the patient was not pregnant, the level of human chorionic gonadotropin was 92 (normal < 5) U/L, which raised concerns about parathyroid carcinoma. However, subsequent single-photon emission computed tomography and parathyroid scan confirmed parathyroid adenoma (Figure 4). The bone lesions seen on imaging were thought to be secondary to osteitis fibrosa cystica, a complication of primary hyperparathyroidism. The patient had no family history of hyperparathyroidism or endocrine diseases.

The patient was referred to endocrinology and then to surgery. She underwent exploration of the parathyroid and excision of a right superior parathyroid adenoma. Biopsy of the left inferior parathyroid gland showed a normal appearance. The left superior and right inferior parathyroid gland were both visualized and appeared normal. The patient returned to the recovery room and then the general ward in good condition.

Intraoperatively, parathyroid hormone fell to $25.3 \mathrm{pmol} / \mathrm{L}$, and the next day it was $1.6 \mathrm{pmol} / \mathrm{L}$, with human chorionic gonadotropin of $11 \mathrm{U} / \mathrm{L}$. The pathology report confirmed parathyroid adenoma, with a minority of the cells $(1 \%)$ staining for human chorionic gonadotropin.

The patient was discharged on the third postoperative day, and the postoperative course was complicated by a full year of severe bone hunger. Four months after surgery, the patient's calcium level was low, at $1.46 \mathrm{mmol} / \mathrm{L}$, despite $2400 \mathrm{~g}$ of oral elemental calcium daily and $0.25 \mu \mathrm{g}$ (10 IU) of 1,25-dihydroxyvitamin $\mathrm{D}_{3}$ three times daily. After $1250 \mu \mathrm{g}(50000 \mathrm{IU})$ vitamin $\mathrm{D}_{2}$ daily was added to the regimen, calcium levels improved to $1.67 \mathrm{mmol} / \mathrm{L}$. One full year after surgery, the

\section{- KEY POINTS}

- When osteolytic lesions are seen on radiography, measurement of serum calcium, albumin, phosphate and parathyroid hormone, along with serum protein electrophoresis, may help to differentiate metabolic bone disease from malignancy.

- Vitamin D deficiency may increase the likelihood of skeletal lesions in primary hyperparathyroidism.
Competing interests: None declared.

The authors have obtained patient consent.

This article has been peer reviewed.

Correspondence to: Jennifer Ringrose, jennifer.ringrose@ualberta.ca

CMAJ 2014. DOI:10.1503 /cmaj.111540 
parathyroid hormone level remained elevated, at $20.0 \mathrm{pmol} / \mathrm{L}$, calcium had normalized to $2.14 \mathrm{mmol} / \mathrm{L}$, and alkaline phosphatase was $180 \mathrm{U} / \mathrm{L}$. About a year after surgery, the 1,25dihydroxyvitamin $\mathrm{D}_{3}$ supplementation was discontinued, and the dosage of vitamin $\mathrm{D}_{2}$ was decreased to $1250 \mu \mathrm{g}$ (50 $000 \mathrm{IU})$ twice weekly. She continued the calcium supplementation. Human chorionic gonadotropin was undetectable at that time.

Almost two years after the surgery, levels of parathyroid hormone, calcium and alkaline phosphatase were all within normal limits. The patient was lost to follow-up until the following year, after the birth of her second child, when her 25-hydroxyvitamin D level was $77 \mathrm{nmol} / \mathrm{L}$ (indi-

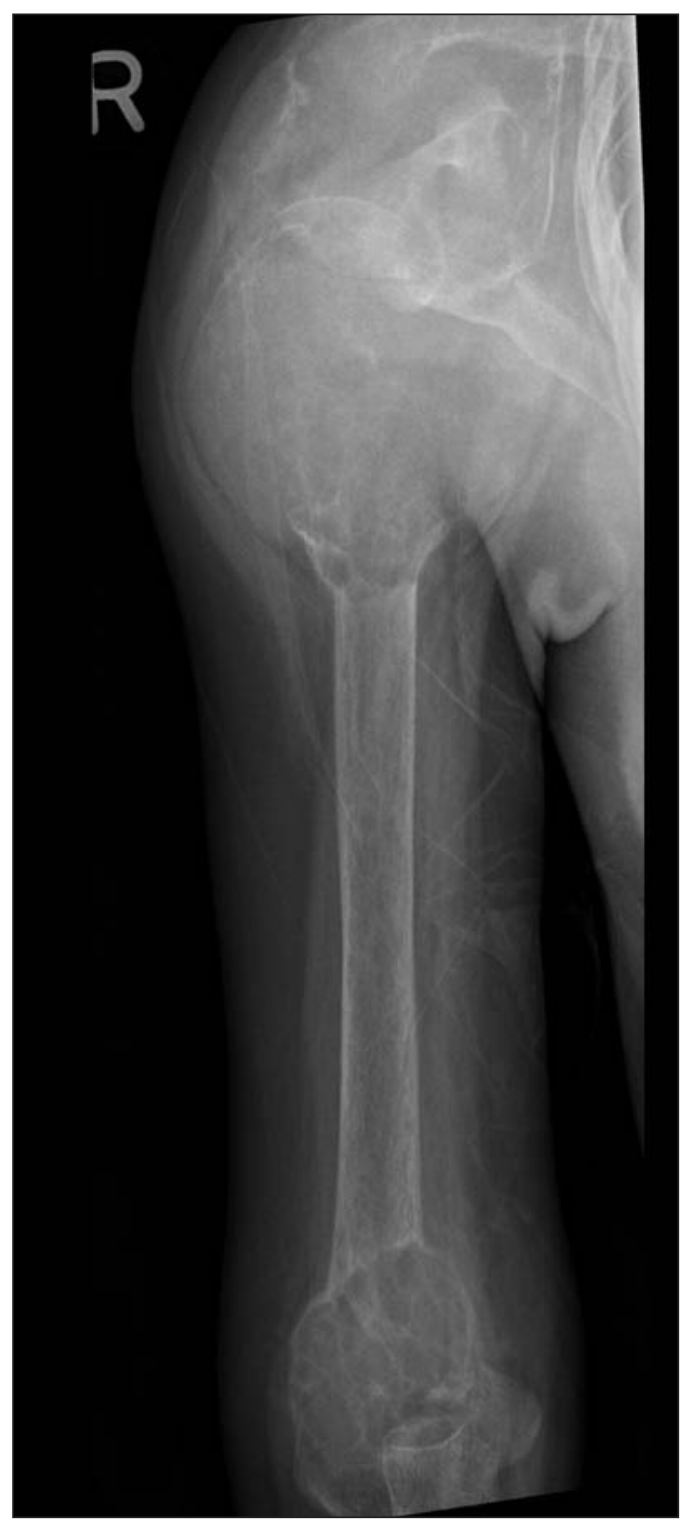

Figure 1: Radiograph of the right humerus of a 21year-old woman. Large lytic lesions are apparent in the humeral head, neck and distal metaphysis and in the scapula. cating mild to moderate deficiency); calcium and parathyroid hormone levels remained normal. Follow-up radiography over the next few years continued to show residual deformities (expansile lesions) from the previously active metabolic bone disease. However, there was some regression relative to the preoperative films.

\section{Discussion}

We have presented a case of primary hyperparathyroidism that initially came to medical attention upon discovery of an expansile bone lesion, originally thought to represent malignancy. The eventual diagnosis was parathyroid adenoma complicated by osteitis fibrosa cystica. The dramatic radiologic presentation, a consequence of severe hypercalcemia and substantially elevated parathyroid hormone, was likely exacerbated by superimposed vitamin $\mathrm{D}$ deficiency.

\section{Osteitis fibrosa cystica}

Osteitis fibrosa cystica is a complication of primary hyperparathyroidism. It is characterized by generalized skeletal demineralization, subperiosteal bone resorption, and the presence of brown tumours and bone cysts. ${ }^{1}$ Fibrovascular replacement of bone marrow may also be seen. ${ }^{1}$

The annual incidence of primary hyperparathyroidism in the United States is 22 cases per 100000 people. $^{2}$ Osteitis fibrosa cystica has been estimated to occur in less than $2 \%$ of cases of primary hyperparathyroidism. ${ }^{3}$ Osteitis fibrosa cystica is rarely seen in developed countries, likely because of the widespread availability of routine blood chemistry testing and thus early identification of the elevated serum calcium levels associated with hyperparathyroidism. ${ }^{1}$ In developing countries, symptomatic hyperparathyroidism predominates. ${ }^{2}$ Patients in these countries may present at a younger age and with more severe bone disease than is the case in developed countries. ${ }^{4}$

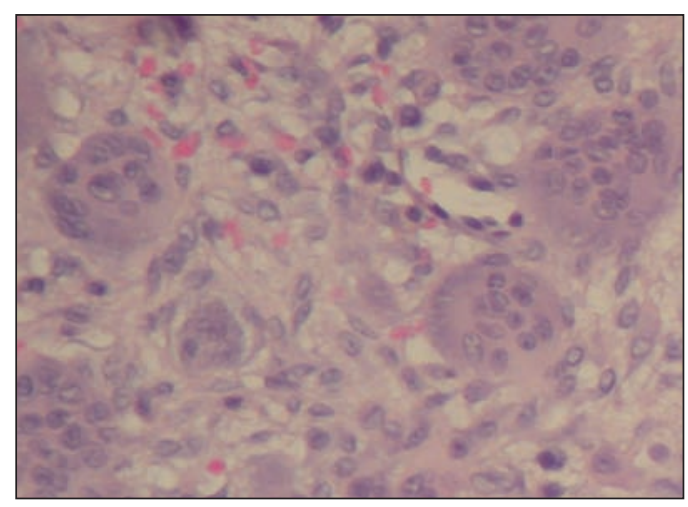

Figure 2: Biopsy specimen of the humeral lesion shows giant cells (hematoxylin and eosin; original magnification $\times 100$ ). 
It can be difficult to differentiate metabolic bone disease from malignant lesions on imaging, and brown tumours of hyperparathyroidism and giant cell tumour of bone may be indistinguishable on histologic examination. This was the situation for our patient at the time of initial bone biopsy. The authors of a similar case report, in which primary hyperparathyroidism mimicked metastatic bone disease, suggested measuring serum calcium, phosphate and parathyroid hormone and performing serum protein electrophoresis before completing further radiologic investigations in patients with multiple osteolytic lesions. ${ }^{5}$

The cure for symptomatic primary hyperparathyroidism is parathyroidectomy, but there are no formal guidelines for the specific treatment of osteitis fibrosa cystica. A recent review of primary hyperparathyroidism suggested maintaining 25hydroxyvitamin $\mathrm{D}$ levels above $50 \mathrm{nmol} / \mathrm{L}$ and ensuring adequate calcium intake in patients with asymptomatic hyperparathyroidism, given that parathyroid overactivity may be exacerbated by low levels of 25-hydroxyvitamin D and low calcium intake. ${ }^{2}$ As for people without hyperparathyroidism, it seems reasonable to aim for vitamin D sufficiency, normocalcemia and adequate calcium intake after parathyroidectomy to achieve maximal regression of bone lesions in osteitis fibrosa cystica.

The long-term prognosis of osteitis fibrosa cystica following parathyroidectomy is difficult to predict. In the patient described here, followup plain radiography showed some regression of the lesions. Others have reported similar regression with calcium and vitamin D replacement. When last measured, our patient's vitamin D level was in the mild to moderate deficiency range, which might delay further regression.

\section{Vitamin D deficiency and hyperparathyroidism}

The patient described here had severe vitamin D deficiency (initial 25-hydroxyvitamin D $9 \mathrm{nmol} / \mathrm{L}$ ) in addition to her primary hyper- parathyroidism. It has been proposed that superimposition of vitamin D deficiency on primary hyperparathyroidism may contribute to the development of skeletal lesions, ${ }^{1}$ as was seen in this patient. Primary hyperparathyroidism has been associated with 25 -hydroxyvitamin D deficiency. In the setting of concomitant 25-hydroxyvitamin D deficiency, higher levels of parathyroid hormone and calcium, more severe disease and higher markers of bone turnover, such as alkaline phosphatase, have been seen. ${ }^{7}$ With concomitant vitamin D deficiency, there is also an increased risk of hungry bone syndrome following parathyroidectomy, ${ }^{8}$ as was experienced by our patient.

This association between low levels of 25hydroxyvitamin D and primary hyperparathyroidism can be described on two levels. First, the disease is more severe in those with concomitant vitamin D deficiency. ${ }^{8}$ Second, vitamin D insufficiency and deficiency are more prevalent among patients with primary hyperparathyroidism than among geographically matched controls. ${ }^{8}$

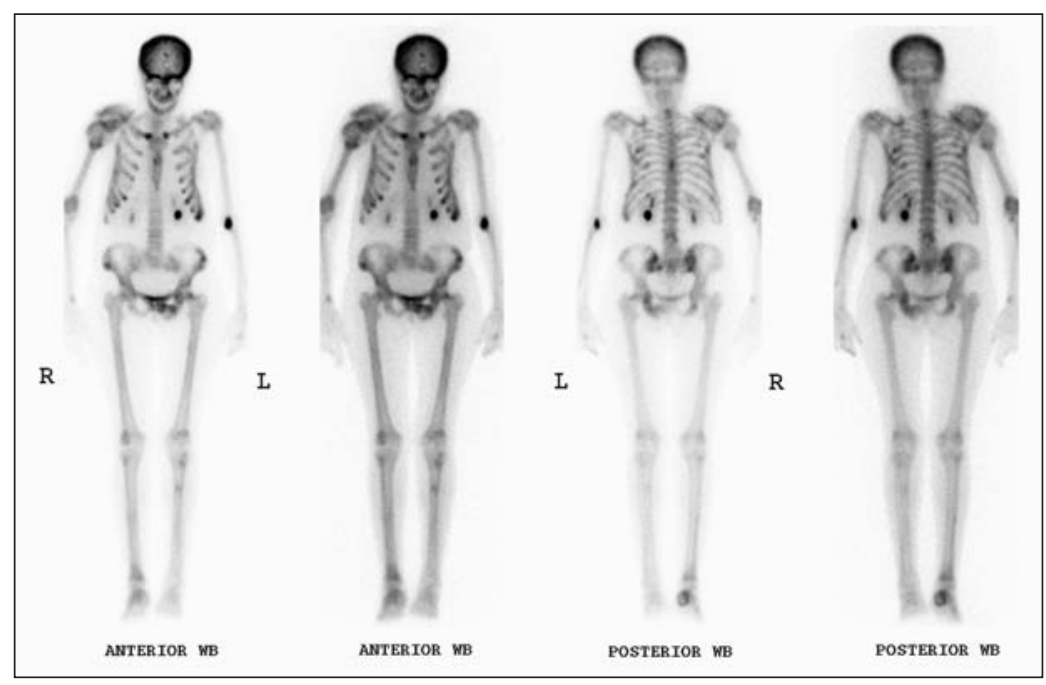

Figure 3: Bone scan showing disseminated multifocal cold lesions superimposed on a diffuse pattern of metabolic bone disease (increased calvarial uptake, prominence of costochondral junctions and subtle increase in the ratio of bone to soft-tissue activity). WB = whole body.

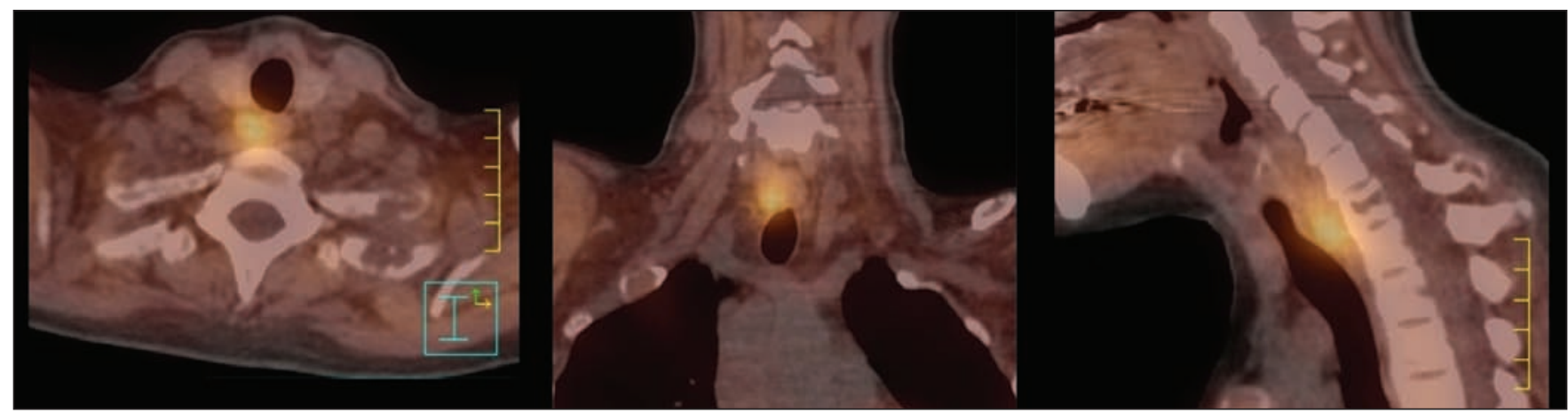

Figure 4: Single-photon emission computed tomography shows a solitary active parathyroid adenoma directly posterior to the trachea and right lobe of the thyroid. Because of its unusual location (posterior rather than inferior to the right thyroid lobe), the adenoma was not clearly visible on conventional planar images. It was precisely localized with this mode of imaging. 
Although the mechanism for these described associations remains to be elucidated, some theories have been proposed. One is that 25 -hydroxyvitamin D deficiency could lead to autonomous parathyroid stimulation, resulting in parathyroid hyperplasia and eventual growth of an adenoma. ${ }^{8}$ Alternatively, chronic vitamin D deficiency could accelerate the growth of a pre-existing adenoma. ${ }^{8}$ Finally, increased 1,25-dihydroxyvitamin D in primary hyperparathyroidism could influence overall vitamin D homeostasis by one of the following mechanisms: inhibition of vitamin $\mathrm{D}$ production in the skin, inhibition of 25-hydroxyvitamin D synthesis in the liver, increased conversion of 25hydroxyvitamin $\mathrm{D}$ to 1,25 -dihydroxyvitamin $\mathrm{D}$ in the kidney or a shortened half-life of 25-hydroxyvitamin $\mathrm{D}$ in primary hyperparathyroidism. ${ }^{8}$

\section{Pregnancy and hyperparathyroidism}

In the case reported here, the relative severity of the bone disease at the time of presentation may have reflected an effect of pregnancy. Pregnancy alters calcium and vitamin D levels: more specifically, fetal demands for calcium lead to increased maternal intestinal absorption of this element, a process mediated by an increased level of 1,25-dihydroxyvitamin $\mathrm{D} .{ }^{9}$ This increase in 1,25-dihydroxyvitamin $\mathrm{D}$ has been attributed to upregulation of 1- $\alpha$ hydroxylase in the maternal kidney, with possible additional contributions from the placenta, decidua and fetal kidneys. ${ }^{9}$ Our patient had severe 25hydroxyvitamin D deficiency, which might have contributed to the severity of her biochemical and bone complications at the time of diagnosis. Other maternal complications of hyperparathyroidism in pregnancy include nephrolithiasis, pancreatitis, change in mental status and hypercalcemic crisis.

Little is known about the outcome of the pregnancy in this case, other than the infant's small size for gestational age. Fetal complications associated with hyperparathyroidism in pregnancy include spontaneous abortion, intrauterine growth restriction, low birth weight, preterm delivery, intrauterine fetal demise and postpartum fetal hypocalcemia. ${ }^{10}$

\section{Delay in diagnosis}

In our patient, the bone complications of primary hyperparathyroidism (i.e., osteitis fibrosa cystica) were evident at initial presentation; however, definitive diagnosis and treatment took one year. Multiple factors likely contributed to this delay in diagnosis.

Primary hyperparathyroidism is most commonly diagnosed because of symptoms of hypercalcemia or when asymptomatic hypercalcemia is discovered on routine blood chemistry. ${ }^{11}$ However, in younger patients, less common presentations may contribute to delays in diagnosis. A review of patients under 30 years of age with hyperparathyroidism showed that younger patients experienced less bone pain and fewer neuropsychiatric symptoms than older patients. ${ }^{12}$ Another case report indicated that infrequent blood tests and vague symptoms may contribute to delayed diagnosis in children. ${ }^{5}$

The patient described here was somewhat reticent in seeking and maintaining medical attention. In addition, biochemical correlation for the pain and lesions seen on initial radiography was delayed. In particular, serum calcium was not measured until one year after the initial presentation. In retrospect, the ureteric stone was probably a complication of the hypercalcemia. Measurement of serum calcium at the time the stone was diagnosed might have helped in making the diagnosis earlier. The elevated alkaline phosphatase was perhaps attributed to the patient's pregnancy, but might have signalled the need to measure serum calcium, phosphate, parathyroid hormone and 25-hydroxyvitamin D.

\section{References}

1. Demay MB, Rosenthal DI, Deshpande V. Case records of the Massachusetts General Hospital. Case 16-2008. A 46-year-old woman with bone pain. N Engl J Med 2008;358:2266-74.

2. Marcocci C, Cetani F. Primary hyperparathyroidism. N Engl J Med 2011;365:2389-97.

3. Gupta A, Horattas MC, Moattari AR, et al. Disseminated brown tumors from hyperparathryroidism masquerading as metastatic cancer: a complication of parathyroid carcinoma. Am Surg 2001; 67:951-5.

4. Mishra SK, Agarwal G, Kar DK, et al. Unique clinical characteristics of primary hyperparathyroidism in India. Br J Surg 2001;88:708-14.

5. Su AW, Chen CF, Huang CK, et al. Primary hyperparathyroidism with brown tumor mimicking metastatic bone malignancy. J Chin Med Assoc 2010;73:177-80.

6. Arabi A, Khoury N, Zahed L, et al. Regression of skeletal manifestations of hyperparathyroidism with oral vitamin D. J Clin Endocrinol Metab 2006;91:2480-3.

7. Eastell R, Arnold A, Brandi ML, et al. Diagnosis of asymptomatic primary hyperparathyroidism: proceedings of the third international workshop. J Clin Endocrinol Metab 2009;94:340-50.

8. Silverberg SJ. Vitamin D deficiency and primary hyperparathyroidism. J Bone Miner Res 2007;22(Suppl 2):V100-4.

9. Chamarthi B, Greene MF, Dluhy RG. Clinical problem-solving. A problem in gestation. N Engl J Med 2011;365:843-8.

10. Schnatz PF, Curry SL. Primary hyperparathyroidism in pregnancy: evidence-based management. Obstet Gynecol Surv 2002;57:365-76.

11. Sitges-Serra A, Bergenfelz A. Clinical update: sporadic primary hyperparathyroidism. Lancet 2007;370:468-70.

12. Cupisti K, Raffel A, Dotzenrath C, et al. Primary hyperparathyroidism in the young age group: particularities of diagnostic and therapeutic schemes. World J Surg 2004;28:1153-6.

Affiliations: Department of Medicine, Division of General Internal Medicine (Ringrose), Department of Radiology and Diagnostic Imaging (Jen), Department of Laboratory Medicine and Pathology (O'Hara) and Department of Medicine, Division of Endocrinology (Toth), University of Alberta, Edmonton, Alta.

Contributors: Dr. Ringrose drafted the manuscript and coordinated all revisions. Dr. Toth assisted with editing the manuscript, particularly the endocrinology-related content, and was directly involved in the patient's care. Dr. Jen assisted with editing the manuscript, provided expert advice regarding the radiology-related content and provided the radiologic images. Dr. O'Hara assisted with editing the manuscript, provided expert opinion regarding pathology-related content and provided the pathological image. All authors approved the final manuscipt and agree to act as guarantors of the work. 